\title{
Electrochemical Performances of SDS-Modified PPy as Supercapacitor Electrode
}

\author{
Yong Chen ${ }^{a,{ }^{*}, \text { Guiying Kang }}{ }^{b}$ \\ College of Perochemical Engineering, Lanzhou University of Technology, Lanzhou 730050, P.R. \\ China \\ a email: yongchen2003@126.com \\ bemail:18193117856@163.com
}

*Corresponding author. Tel: +86931 7823115

Keywords: supercapacitor; polypyrrole; sodium dodecyl sulfonate.

Abstract.Conducting polypyrrole (PPy) is an attractive class of electrode material to be used in supercapacitor. However, the main chain of PPy prone to rupture in the process of charging and discharging, which results in premature attenuation and decreasing of specific capacitance. The SDS-modified PPy is prepared by the chemical oxidation method for improving specific capacitance of PPy and synthesis conditions are optimized through single factor way. Meanwhile, the optimal conditions are obtained, including the temperature of polymerization $\left(0^{\circ} \mathrm{C}\right)$, the time of polymerization (4h), the molar ratio of Py/SDS $\left(n_{\mathrm{Py}}: n_{\mathrm{SDS}}=4: 1\right)$ and APS/Py $\left(n_{\mathrm{APS}}: n_{\mathrm{Py}}=1.3: 1\right)$, and the concentration of electrolyte $\left(0.5 \mathrm{~mol} \mathrm{~L}^{-1} \mathrm{Na}_{2} \mathrm{SO}_{4}\right)$. A three-electrode cell system is used to evaluate the electrochemical performances of PPy/SDS by cyclic voltammetry, galvanostatic charge/discharge, and electrochemical impedance at room temperature. The results showed that PPy/SDS has higher specific capacitance than that of pure PPy. Besides, the capacity retention rate of PPy/SDS is higher than that of PPy.

\section{Introduction}

In conventional capacitor, electrical energy is stored by the removal of electrons from one metal plate to another. This kind of separation of charge creates a potential difference between the two plates and is limited by the dielectric breakdown. Thus, the energy stored in a conventional capacitor depends on the materials property of the plates. Since the 20th century, as supercapacitors can lead to exhibit an unusually high energy density, longer cycle life and the minimum amount of charge separation, it have attracted considerable attention over the past few decades over the conventional capacitor [1-4]. Basically, supercapacitors have two energy storage mechanisms, electrical double-layer capacitance (EDLC) in which the energy is stored using ion adsorption and the pseudocapacitance where energy can be stored by fast surface redox reaction [5-6]. In general, the energy densities of a pseudocapacitance-based device higher as compared to EDLCs [1]. Conducting polymers has been chosen as a supercapacitor electrode material owing to its high electrical conduction and wide application. Such as PolyPyrrole (PPy), Polyaniline (PANI) and Polythiophene (PTh) and their derivatives, especially PPy is widely used because of its easy synthesis, high electrical conductivity and low cost [7-8]. Pure PPy as a supercapacitor electrode has lower specific capacitance and cycle stability. So, the SDS-modified PPy was prepared, the sodium dodecyl sulfonate has been chosen both as dopant and surfactant for the chemical oxidative polymerization of pyrrole to improve the stability and specific capacitance of PPy. Its electrochemical performance was investigated by three-electrode cell system. The results showed that PPy/SDS has higher specific capacitance and cycle stability than that of pure Py.

\section{Experimental section}

\subsection{Materials}

Pyrrole monomer, having a molar mass of $67 \mathrm{~g} \mathrm{~mol}^{-1}$ and density of $0.97 \mathrm{~g} \mathrm{~cm}^{-3}$, was purchased from 
factory of Tianjin chemical reagent. Sodium dodecyl sulfonate (dopant and surfactant), Ammonium peroxodisulfate (oxidizing agent), and Anhydrous sodium sulfate (electrolyte solution) were purchased from three factory of Shanghai reagent. All other chemicals were used as received, without any further purification. Distilled water was used throughout the whole preparation process.

\subsection{Syntheses of SDS-modified PPy}

Firstly, SDS (7.229mmol) was sonicated for $10 \mathrm{~min}$ in $150 \mathrm{~mL}$ of distilled water for dissolving. Then, distilled pyrrole $(1 \mathrm{~mL}, 14.458 \mathrm{mmol})$ was added to the solution and the whole solution was stirred at room temperature for $2 \mathrm{~h}$. Secondly, APS $(14.458 \mathrm{mmol})$ was dissolved in $50 \mathrm{~mL}$ of distilled water in a beaker and the solution was added drop-wise to the mixture of SDS and Py, the polymerization started immediately, and the reaction was allowed to continue for $4 \mathrm{~h}$ under the vigorous stirring and the $0{ }^{\circ} \mathrm{C}$ of reactive temperature. Finally, the resulting PPy was washed with distilled water and ethanol until the filtrate become colorless. And then the product was dried at $60{ }^{\circ} \mathrm{C}$ for $24 \mathrm{~h}$.

\section{Results and discussion}

\subsection{FTIR analysis}

FTIR has been accomplished to assess the chemical environment of the PPy/SDS, is shown in Fig. 1. The peaks at $1040,1550,1460$, and $3430 \mathrm{~cm}^{-1}$, respectively, are due to $\mathrm{C}-\mathrm{H}, \mathrm{C}=\mathrm{C}, \mathrm{C}-\mathrm{N}$, and $\mathrm{N}-\mathrm{H}$ stretching vibration of the PPy ring, and the peak at $1300 \mathrm{~cm}^{-1}$ correspond to $\mathrm{C}-\mathrm{C}$ backbone stretching of Ppy[9]. Besides, the peaks at 912, 806, and $677 \mathrm{~cm}^{-1}$ are designated to the $\mathrm{C}-\mathrm{H}$ out-plane vibration of the PPy. In the image of PPy/SDS, the peak at $2920 \mathrm{~cm}^{-1}$ attributed to the vibration of $-\mathrm{CH}_{3}$ of the SDS which prove the SDS existing in the PPy backbone. It can be also find that the peak at $1700 \mathrm{~cm}^{-1}$ which from the vibration of carbonyl.

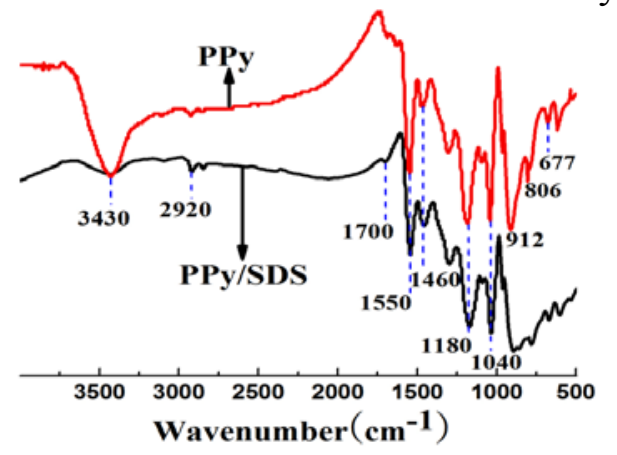

Fig. 1 FTIR spectra of PPy and PPy/SDS

\subsection{Electrochemical studies}

\subsubsection{Influencing factors of specific capacitance of PPy/SDS}

The process of synthesis conditions was optimized by single factor way. It can find those factors that influencing the specific capacitance of PPy/SDS from Fig. 2. Meanwhile, the highest specific capacitance of PPy/SDS was obtained under the optimal conditions that $0^{\circ} \mathrm{C}$ of reactive temperature, $n(\mathrm{Py}): n(\mathrm{SDS})=4: 1, n(\mathrm{APS}): n(\mathrm{Py})=1.3: 1$, four hours of reactive time and $0.5 \mathrm{~mol} \mathrm{~L}^{-1} \mathrm{Na}_{2} \mathrm{SO}_{4}$ electrolyte. 

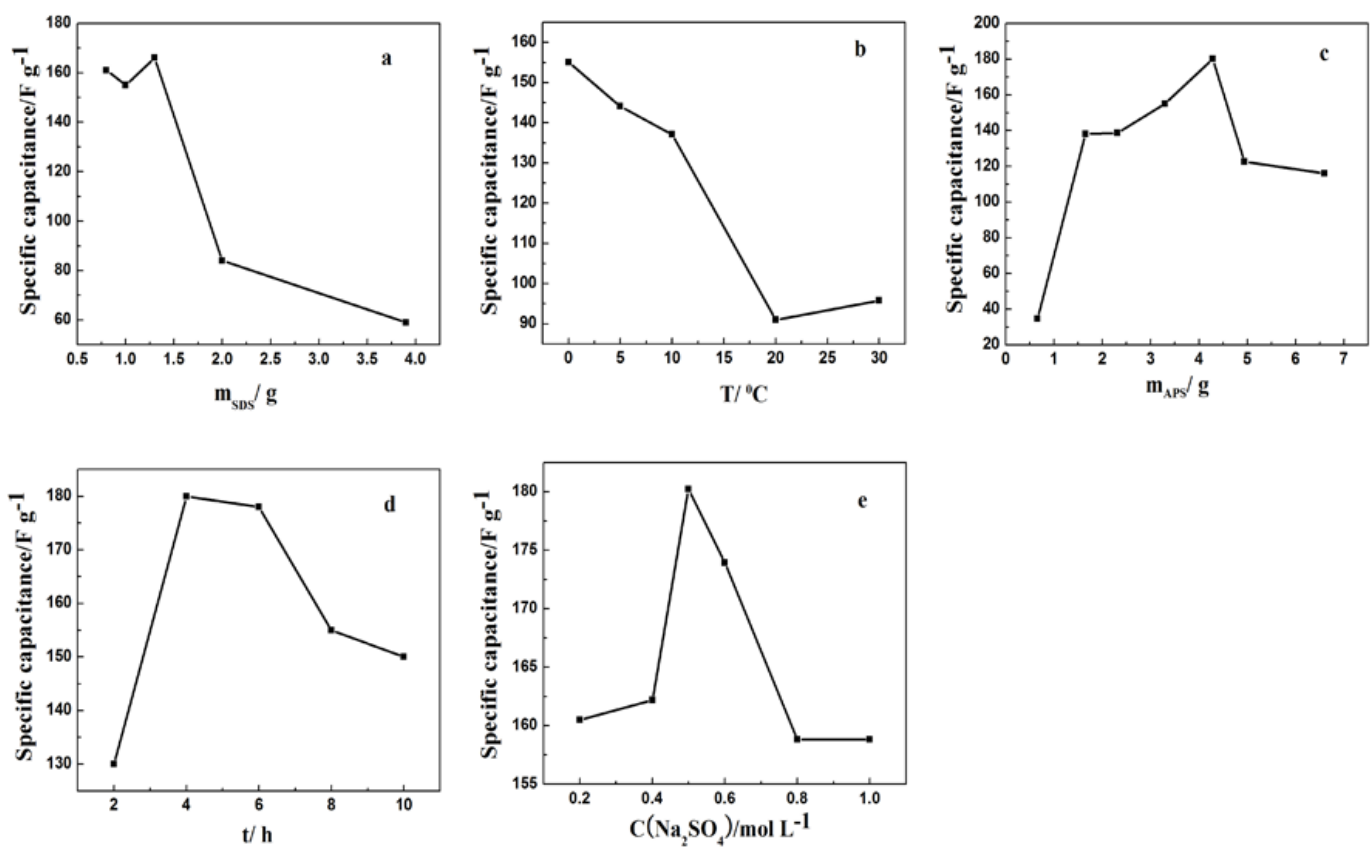

Fig. 2 (a), (b), (c), (d) and (e) are variations in the specific capacitance of PPy/SDS as a function of quality of SDS, reactive temperature, quality of APS, reactive time and concentration of $\mathrm{Na}_{2} \mathrm{SO}_{4}$, respectively.

\subsubsection{Cyclic Voltammetry}

It can be found that the SDS-modified PPy has higher specific capacitance than that of pure PPy, which is confirmed from the area of cyclic voltammetry curve (Fig. 3a). The redox peaks of PPy/SDS can be easily observed, under the same scanning rate. Therefore, the PPy/SDS has good electrochemical reversibility according to the redox peaks.

Fig. $3 b$ shows the specific capacitance of PPy/SDS and pure PPy at different scanning rate. Specific capacitance $(\mathrm{C})$ of the materials is calculated from CV measurements using the following equation[10]:

$\mathrm{C}=\left(\frac{\mathrm{i}}{\mathrm{m} \times \mathrm{dv} / \mathrm{dt}}\right)$

where $\mathrm{i}$ is the average current in the capacitive potential region $(-0.6 \sim 0.8 \mathrm{~V}), \mathrm{dv} / \mathrm{dt}$ is the scan rate and $\mathrm{m}$ is the mass of the material.

The high value of specific capacitance of PPy and PPy/SDS at a lower scanning rate owing to the oxidation or deoxidation of $\alpha-\mathrm{C}$ or $\beta-\mathrm{C}$ atoms, and the ions get enough time to migrate freely in the PPy matrix[11].
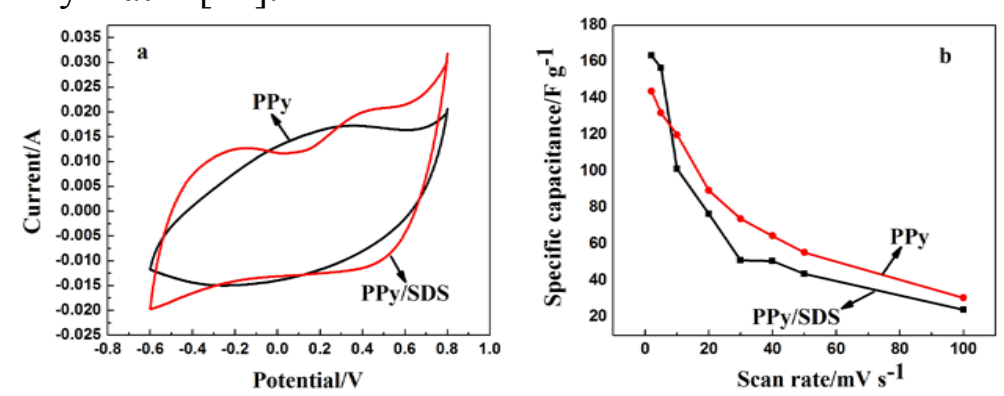

Fig. 3 (a) CV curves of PPy/SDS and PPy at a scan rate of $10 \mathrm{mV} \mathrm{s}^{-1}$; and (b) variation in the specific capacitance of PPy/SDS and PPy as a function of scan rate

\subsubsection{Galvanostatic charge-discharge}

In order to evaluate the charge storage capacity, the galvanostatic charge-discharge analysis has been performed at a current density of $5 \mathrm{~mA} \mathrm{~cm}^{-2}$ (Fig. 4a). Specific capacitance (C) of the materials is calculated from GCD measurements using the following equation: 


$$
\mathrm{C}=\frac{\mathrm{I} \Delta \mathrm{t}}{\mathrm{m} \Delta \mathrm{V}}
$$

Where $\mathrm{I}(\mathrm{A})$ is the discharge current, $\Delta \mathrm{t}(\mathrm{s})$ is the discharge time, $\Delta \mathrm{V}(\mathrm{V})$ is the capacitive potential region $(-0.6 \sim 0.8 \mathrm{~V})$ and $\mathrm{m}(\mathrm{g})$ is the mass of the active material.

The specific capacitance values of pure PPy and PPy/SDS, are calculated to be 136, and $180 \mathrm{~F} \mathrm{~g}^{-1}$, respectively. It indicates that SDS-modified PPy can enhance the specific capacitance of PPy.

Fig. $4 \mathrm{~b}$ shows that the specific capacitance of PPy/SDS is increased at lower current density. The reason is that the ion in the electrolyte can easily diffuse to the surface and interior of electrode material at lower current density[9].
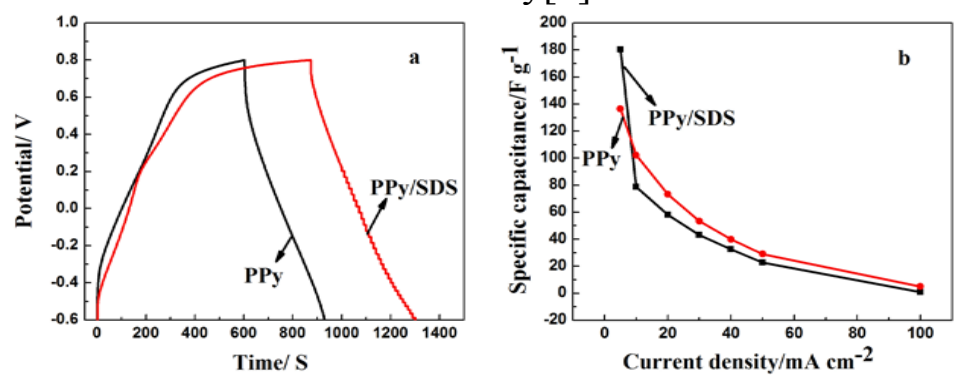

Fig. 4 (a) GCD curves of PPy/SDS and PPy at a current density of $5 \mathrm{~mA} \mathrm{~cm}^{-2}$; and (b) variation of specific capacitance of PPy/SDS and PPy as a function of current density.

\subsubsection{Electrochemical impedance}

Fig. 5a shows that the resistance of charge transfer and the diffusion impedance of PPy/SDS are lower than that of pure PPy, and their resistance of solution is similar. The reason is probably due to the SDS was doped in the backbone of PPy, which enhance the delocalization of electron, extend the conjugated-system of p-electron and enlarge the moving range. So, the process of charge transfer can proceed easily. The PPy and PPy/SDS as an electrode have been subjected to 100 cycles at the current density of $2 \mathrm{~A} \mathrm{~g}^{-1}$. It can be found that the cycle stability of PPy/SDS better than that of PPy (Fig. 5b).
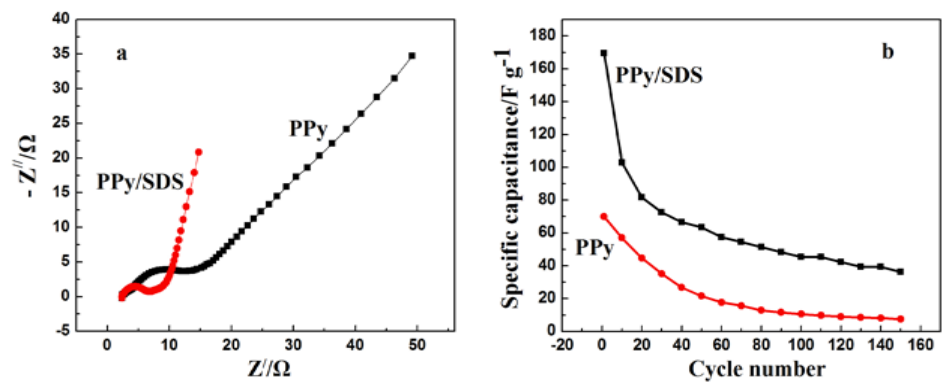

Fig. 5 (a) Electrochemical impedance of PPy/SDS and PPy at opening circuit voltage; and (b) Cycle stability tests of PPy/SDS and PPy at the current density of $2 \mathrm{~A} \mathrm{~g}^{-1}$.

\section{Conclusions}

The introduction of SDS changes the backbone of PPy, which provides a large surface area for diffusion of ion in the electrolyte and electrode, and hence facilitates electrochemical utilization of PPy. Meanwhile, the specific capacitance of PPy can be also improved. Therefore, these structural features make the PPy/SDS to be a possible and promising electrode material for supercapacitor.

\section{References}

[1] Stoller MD, Park S, Zhu Y, An J, Ruoff RS. Nano Letters. 2008;8:3498-502.

[2] Chang J-K, Huang C-H, Tsai W-T, Deng M-J, Sun I-W. Journal of Power Sources. 2008; 179:435-40. 
[3] An G, Yu P, Xiao M, Liu Z, Miao Z, Ding K, et al. Nanotechnology. 2008;19:275709.

[4] Liu D, Zhang Q, Xiao P, Garcia BB, Guo Q, Champion R, et al. Chemistry of Materials. 2008;20:1376-80.

[5] Simon P, Gogotsi Y. Nat Mater. 2008;7:845-54.

[6] Raymundo-Pinero E, Khomenko V, Frackowiak E, Beguin F. Journal of the Electrochemical Society. 2005;152:A229-A35.

[7] Wu T-M, Chang H-L, Lin Y-W. Composites Science and Technology. 2009;69:639-44.

[8] Wu TM, Lin SH. Journal of Polymer Science Part A: Polymer Chemistry. 2006;44:6449-57.

[9] Singh AK, Sarkar D, Khan GG, Mandal K. Journal of Materials Chemistry A. 2013;1:12759-67.

[10] Fan L-Z, Maier J. Electrochemistry communications. 2006;8:937-40.

[11] Jiang F, Zhou T, Tan S, Zhu Y, Liu Y, Yuan D. Int J Electrochem Sci. 2009;4:1541-7. 